\title{
Mapping of the interaction sites between Wee1 kinase and the regulatory $\beta$-subunit of protein kinase CK2
}

\author{
BIRGITTE B. OLSEN ${ }^{1}$, JAN N. KREUTZER ${ }^{1}$, NOBUMOTO WATANABE ${ }^{2}$, \\ TINA HOLM ${ }^{1}$ and BARBARA GUERRA ${ }^{1}$ \\ ${ }^{1}$ Institute of Biochemistry and Molecular Biology, University of Southern Denmark, Odense, Denmark; \\ ${ }^{2}$ Department of Chemical Biology, Advanced Research Institute (ASI), \\ RIKEN 2-1, Hirosawa, Wako, Saitama 351-0198, Japan
}

Received November 27, 2009; Accepted January 12, 2010

DOI: 10.3892/ijo_00000600

\begin{abstract}
Somatic Wee1 is a protein kinase that plays a key role in cell cycle progression at the onset of mitosis by phosphorylating CDK1 at the inhibitory Tyr15 amino acid residue. Wee1 is regulated at multiple levels, i.e., phosphorylation, protein-protein association and proteasome-mediated degradation. We have recently shown that the regulatory ß-subunit of protein kinase CK2 participates in PLK1-Wee1 complex formation interacting directly with Wee1 and thereby contributing to the regulation of G2-M cell cycle transition. Here, we show that Wee 1 binds CK2B via two domains comprising amino acids 59-71 and 232-332. By employing deletion mutants of the CK2ß-subunit we also show that two regions between residues 1-5 and 155-170 are necessary for binding Wee1. Furthermore, we demonstrate that the interaction between $\mathrm{CK} 2 \beta$ and Wee 1 does not modify the kinase activity of the latter, instead CK $2 \beta$ directly upregulates CDK1 kinase activity by reversing the inhibitory effect which follows Wee1-mediated phosphorylation. Taken together, our findings reinforce the notion that CK2B is a modulator of protein kinases implicated in cell cycle regulation and exerts functions that are independent of CK2 tetramers.
\end{abstract}

\section{Introduction}

Protein kinase CK2 is a highly conserved enzyme ubiquitously expressed among all the eukaryotic organisms investigated so far. It is composed of two catalytic subunits $\alpha$ and/or $\alpha^{\prime}$ and two regulatory $ß$-subunits. CK2 was originally described as an enzyme characterized by a tetrameric structure but evidence suggests that the individual subunits can exist in

Correspondence to: Dr Barbara Guerra, Department of Biochemistry and Molecular Biology, University of Southern Denmark, Campusvej 55, 5230 Odense, Denmark

E-mail: bag@bmb.sdu.dk

Key words: CK2, Wee1, CDK1, protein association, phosphorylation association with various intracellular proteins $(1,2)$. Because of the broad spectrum of proteins that interact with or are phosphorylated by CK2, it is not surprising that this enzyme is implicated in various intracellular processes ranging from regulation of transcription, DNA replication and repair to survival, proliferation and differentiation (reviewed in refs. 2,3). Numerous studies have also suggested that CK2 exerts a vital function. Indeed it was shown by gene knockout experiments in mammals that both the $\alpha$ - and $\beta$-subunits are essential for the development of mice $(4,5)$ while lack of expression of the $\alpha^{\prime}$-subunit is not lethal but leads to infertility (6). CK2 has also been linked to the regulation of cell cycle progression in lower as well as higher eukaryotes (reviewed in refs. 7,8).

Cell cycle progression is controlled by numerous proteins which act in order to preserve the integrity of the genetic information crucial for the survival of a cell. Entry into mitosis is mainly regulated by CDK1 whose optimal activation is achieved following the association with cyclin B and phosphorylation in the conserved T-loop domain. During interphase, CDK1 activity is maintained low by phosphorylation of two amino acids residues (i.e., Thr14 and Tyr15) catalyzed by Myt1 and Wee1 protein kinases $(9,10)$. Entry into mitosis requires the intervention of the $\mathrm{CDC} 25$ dual specificity protein phosphatase family members that catalyze the activating dephosphorylation of CDK1 at the aforementioned amino acid residues (reviewed in ref. 11).

Previously, we have shown that the regulatory $\beta$-subunit of CK2 interacts with and up-regulates the checkpoint kinase Chk1 (12) and that the overexpression of CK2ß leads to accelerated degradation of CDC 25 mediated by Chk1 (13). More recently, we have revealed a further regulatory contribution of CK $2 \beta$ to the G2/M cell cycle transition through the modulation of Wee1 expression. Wee1 activity rises during the $\mathrm{S}$ and $\mathrm{G} 2$ phases of the cell cycle and declines at the onset of mitosis when full activation of CDK1 is required. The stability of Wee1 is regulated by the CDK1/cyclin B-mediated phosphorylation at Ser123 which creates a phosphodegron recognized by the $\beta$-transducin repeat-containing protein $(\beta-\mathrm{TrCP})$ and a docking site for the binding of the polo-like kinase 1 [PLK1, $(14,15)]$. We found that CK2ß participates in the PLK1-Wee1 complex formation and interacts directly with Wee1 (16). 
In the present study, we have attempted to narrow down the binding regions of Wee1 as well as of the regulatory B-subunit of protein kinase CK2. Furthermore, by employing selected CK $2 \beta$ constructs we show that CK2 $2 \beta$ does not modify the kinase activity of Wee1, instead, increasing amounts of CK2B directly up-regulate the kinase activity of CDK1 in vitro.

\section{Materials and methods}

All experiments indicated in the figures were repeated at least three times obtaining similar results.

Cell culture and reagents. Cos-1 cells were purchased from the American Type Culture Collection and grown in the presence of Dulbecco's modified Eagle's medium (DMEM, Gibco) supplemented with $10 \%$ fetal bovine serum and $1 \mathrm{mM} \mathrm{L}-$ glutamine. Cells were grown at $37^{\circ} \mathrm{C}$ under a $5 \% \mathrm{CO}_{2}$ atmosphere. Transient overexpression of proteins was performed by transfection of plasmid DNA with FuGene 6 reagent (Roche) following the manufacturer's recommendations.

Plasmid constructs. The various CK2 3 cDNAs (except full length and the 1-155 deletion mutant described in ref. 12) have been cloned into the mammalian expression vector pcDNA3.1-MycHis (Invitrogen) using the polymerase chain reaction (PCR) and inserted into BamHI-XhoI restriction sites. The following primers were employed: CK2ß(5-215)MycHis 5'-CGC GGA TCC ATG GAG GAG GTG TCC TGG ATT TCC-3' (forward primer), 5'-CCG CTC GAG G GCG AAT CGT CTT GAC TGG GCT C-3' (reverse primer); CK2ß(72-215)-MycHis 5'-CGC GGA TCC ATG ATT GAG CAG GCA GCC GAG GCG-3' (forward primer), 5'-CCG CTC GAG G GCG AAT CGT CTT GAC TGG GCT C-3' (reverse primer); CK2ß(1-208)-MycHis 5'-CGC GGA TCC ATG AGC AGC TCA GAG GAG GTG-3' (forward primer), 5'-CCG CTC GAG G CTT GAA GTT GCT GGC GGC TTG G-3' (reverse primer); CK2ß(1-193)-MycHis 5'-CGC GGA TCC ATG AGC AGC TCA GAG GAG GTG-3' (forward primer), 5'-CCG CTC GAG G ATG GAT CTT GAA ACC GTA GAG CC-3' (reverse primer); CK2ß(1-170)-MycHis 5'-CGC GGA TCC ATG AGC AGC TCA GAG GAG GTG -3' (forward primer), 5'-CCG CTC GAG G CAC CAT GAA GAG CAT GTG AGG-3' (reverse primer); CK2ß(1-155)-MycHis 5'-CGC GGA TCC ATG AGC AGC TCA GAG GAG GTG-3' (forward primer), 5'-CCG CTC GAG G ATC CGT GTG ATG GTG TCT TG-3' (reverse primer). The correct sequence and orientation were verified by DNA sequencing. The $\mathrm{N}$-terminal Wee1 deletion mutants were as described (14). As for the $\mathrm{C}$-terminal fragments, amino acids indicated in the figures were changed into stop codons.

Preparation of cell extract, Western blot analysis and immunoprecipitation. Cell pellets were re-suspended in cold lysis buffer $(50 \mathrm{mM}$ Tris/ $\mathrm{HCl} \mathrm{pH} 7.5,150 \mathrm{mM} \mathrm{NaCl}, 1 \%$ Triton $\mathrm{X}-100,10 \%$ glycerol, $1 \mathrm{mM}$ DTT, $1 \mathrm{mM} \mathrm{Na} \mathrm{VO}_{4}, 30 \mathrm{mM}$ B-glycerophosphate $10 \mathrm{mM} \mathrm{NaF}$ and $100 \mathrm{nM}$ okadaic acid) containing a protease inhibitor cocktail (Roche). Cells were sonicated and subsequently centrifuged at $4^{\circ} \mathrm{C}$ for $30 \mathrm{~min}$ at $10,000 \mathrm{~g}$. Whole extracts were subjected to SDS-PAGE and subsequently transferred to a PVDF membrane (Bio-Rad).
Protein-antibody complexes were visualized by a chemiluminescence detection system following the manufacturer's guidelines (CDP Star, Applied Biosytems). Cell lysates were also used for immunoprecipitation assays essentially as previously described (17).

Antibodies. The following antibodies were employed: mouse monoclonal anti-Wee1 (B-11, the binding epitope is located in the C-terminal domain), rabbit polyclonal anti-Wee1 (C-20), mouse monoclonal anti-Myc (9E10) and mouse monoclonal anti-CDK1 (all from Santa Cruz Biotechnology); rabbit polyclonal anti-Wee1 (the binding epitopes are in the N-terminal region), rabbit polyclonal anti-phospho-CDK1 (Tyr15), rabbit monoclonal anti-Myc (71D10) and mouse monoclonal anti-Myc (9B11, all from Cell Signaling Technology); mouse monoclonal anti- $\beta$-actin (Sigma); mouse monoclonal antiCK $2 \alpha$ and -CK2ß (both from Calbiochem). Rabbit polyclonal anti-CK2 $2 \beta$ was obtained by immunizing rabbits with the full length protein. Goat polyclonal anti-GST was from GE Healthcare.

Chromatographic analysis of Wee1-CK $2 \beta$ complex formation. Crude extract from Cos- 1 cells co-transfected with various plasmids as indicated in the figure legends, was analyzed by analytical gel filtration chromatography using a Superdex 200 column PC 3.2/30 (SMART System Amersham Biosciences) equilibrated in a buffer containing $25 \mathrm{mM}$ Tris $\mathrm{pH} 8.5,300 \mathrm{mM} \mathrm{NaCl}$ and $1 \mathrm{mM}$ DTT. After chromatography, selected fractions were subjected to immunoprecipitation and Western blot analysis.

Recombinant proteins. Human recombinant $\mathrm{CK} 2 \alpha,-\alpha^{\prime},-\beta$ and CK2ß(1-155) deletion mutant were expressed and purified as previously described (18). Human recombinant Wee1 (N-terminal GST-tag) was purchased from Calbiochem, histone $\mathrm{H} 1$ was from Roche and active recombinant CDK1 in complex with cyclin B was from Millipore.

Kinase assays. In vitro CDK1 kinase assay was performed in the presence of human recombinant CDK1 in complex with cyclin $\mathrm{B}$, histone $\mathrm{H} 1$ as a substrate, Wee1 and increasing amounts of either full length CK2ß or the CK2ß(1-155) deletion mutant as indicated in the figure legends. Reaction mixtures were incubated for $30 \mathrm{~min}$ at $30^{\circ} \mathrm{C}$ in the presence of kinase buffer (50 mM Tris pH 7.4, $10 \mathrm{mM} \mathrm{MgCl}_{2}, 1 \mathrm{mM}$ DTT, $0.1 \mathrm{mM}$ EDTA, $10 \mathrm{mM}$ ß-glycerophosphate, $50 \mu \mathrm{M}$ ATP and $\left.10 \mu \mathrm{Ci}\left[\gamma^{-32} \mathrm{P}\right] \mathrm{ATP}\right)$. Reactions were stopped by adding SDS-sample buffer and subsequently analyzed by SDS-PAGE and Western blotting. Radioactive protein bands were revealed by autoradiography and the incorporated radioactivity was quantified by scintillation counting (Packard) of the excised radioactive protein bands.

\section{Results}

Weel kinase predominantly associates with the regulatory $\beta$-subunit of protein kinase CK2. Mapping of the CK2 $\beta$ binding regions. We have previously reported that Wee1 kinase interacts with CK2ß in cells transiently transfected with Wee1 and either CK2 $\alpha^{\prime}$ or CK2ß plasmids DNA (19). In order to 


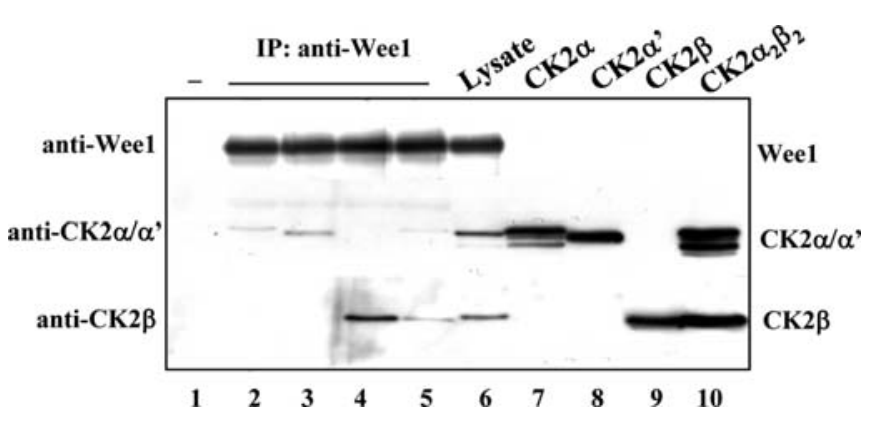

Figure $1 . \mathrm{CK} 2 \beta$ is the major interacting protein of Wee1 kinase in vitro. Whole lysates $(200 \mu \mathrm{g})$ from Cos-1 cells transiently overexpressing Wee1 were mixed with equimolar amounts (16 pmol) of recombinant CK $2 \alpha$ (lane 2), CK2 $\alpha^{\prime}$ (lane 3), CK2ß (lane 4) or CK2 holoenzyme (lane 5). The complexes were subjected to immunoprecipitation (IP) with a rabbit polyclonal anti-Wee1 (C-20) antibody. Lane $1(-)$, is a control experiment where the lysate was combined with 16 pmol recombinant CK2 holoenzyme and the immunoprecipitation was performed with a control serum. Lanes 6-10, show the detection of Wee1 (30 $\mu$ g lysate), CK2 $\alpha$, CK2 $2 \alpha^{\prime}$, CK2 $2 \beta$ and CK2 holoenzyme ( $1 \mathrm{pmol}$ of each recombinant protein), respectively. Proteins were analyzed by Western blotting using the indicated mouse monoclonal antibodies

further characterize the association between CK2 and Wee1, we have conducted in vitro immunoprecipitation experiments (Fig. 1) employing whole extracts from cells transfected with Wee1 plasmid DNA (lanes 1-5), as a source of Wee1 kinase, and equimolar amounts of recombinant purified CK2 $\alpha$ (lane 2), CK2 $\alpha$ ' (lane 3), CK2ß (lane 4) and CK2 holoenzyme (lanes 1 and 5), respectively. As showed in Fig. 1, we confirmed that Wee 1 mainly interacts with the regulatory $ß$-subunit of CK2 (lane 4). Based on the band intensity resulting from the detection of 1 pmol of recombinant CK2ß (lane 9), we concluded that about $0.5 \mathrm{pmol}$ of $\mathrm{CK} 2 ß$ co-immunoprecipitated with Wee1. With respect to the other recombinant proteins, we observed a much weaker interaction with Wee1 estimating that 0.1 (lane 3) or $<0.1$ pmol of proteins (lanes 2 and 5) coimmunoprecipitated with the aforementioned kinase. In order to narrow down the $\mathrm{CK} 2 \beta$ region(s) involved in the interaction with Wee1, we constructed various plasmids coding for either $\mathrm{N}$-terminal or C-terminal CK2ß deletion mutants as indicated in Fig. 2A. Cos-1 cells were then employed to transiently coexpress Wee 1 and either of the indicated CK2 $2 \beta$ constructs. The expression levels of the entire panel of CK2ß deletion mutants analyzed by Western blotting was comparable as shown in Fig. 2B and D. Whole lysates were then subjected to immunoprecipitation employing polyclonal anti-Wee1 antibody. Results shown in Fig. 2C and E indicate that the major region involved in the interaction with Wee1 resides in the C-terminal domain of CK2 $2 \beta$ and it is comprised between amino acids 155 and 170 (Fig. 2E) as mutant CK2ß(1-155)MycHis failed to interact with Wee1. We also noted that the $\mathrm{N}$-terminal domain of CK $2 \beta$ contributes to the association with Wee 1 as lack of 5 amino acid residues (i.e., mutant CK2ß(5-215)-MycHis) resulted in a weaker but still detectable interaction with Wee1 (Fig. 2C).

Identification of Weel amino acid residues mediating the interaction with $C K 2 \beta$. In order to identify the Wee 1 amino acid residues involved in the association with CK2B we employed a panel of plasmid DNA constructs expressing various $\mathrm{N}$ - and $\mathrm{C}$-terminal Wee1 deletion mutants following transient transfection of Cos-1 cells (Fig. 3A). Whole extracts from cells co-transfected with CK2ß-MycHis and either of the individual Wee1 constructs were subjected to Western blot analysis. Since the various $\mathrm{N}$-terminal deletion mutants were not equally expressed (i.e., Fig. 3B, lanes 6 and 7) we attempted to normalize their expression levels prior to proceeding with further assays (Fig. 3B, lower panel). Whole extracts were then subjected to immunoprecipitation with rabbit monoclonal anti-Myc antibody. As shown in Fig. 3C, one of the regions involved in the association with CK2 $2 \beta$ mapped between amino acids 59 and 71 as association with CK2ß was still detectable when we employed the $\Delta$ N59-Wee 1 deletion mutant (lane 5) but not the subsequent one (i.e., $\Delta$ N71-Wee1, lane 6). We performed a similar analysis employing a set of C-terminal Wee1 deletion mutants whose expression levels were compared before proceeding with further experiments (Fig. 3D). Co-immunoprecipitation experiments revealed that a second Wee1 region contributes to the association with $\mathrm{CK} 2 \beta$ that is comprised between amino acids 232 and 332 (Fig. 3E) as the Wee1-332* deletion mutant was still able to associate with CK2ß (lane 7) but not the subsequent Wee1 fragment (i.e., Wee1-232*, lane 8). Thus, from this analysis we could conclude that two regions are equally important for the binding of Wee 1 to CK2B as the absence of either one destabilizes the association between the aforementioned proteins. It is worth noting that for the immunoprecipitation experiments and the detection of the individual proteins by Western blotting, we employed various antibodies according to the location of the epitopes recognized by the anti-Wee1 antibodies and the Wee1 deletion mutants under investigation. As shown in Fig. 3E, Wee1 was detected with rabbit polyclonal antibody which made it possible to also detect the endogenous Wee1 co-precipitated with CK2ß-MycHis. Wee1 was detected with a mouse monoclonal antibody whose epitope maps in the C-terminal domain of Wee1 (Fig. 3C). Under the conditions applied for detecting Wee1, this antibody did not promptly reveal the coprecipitated endogenous full length Wee1.

We attempted to confirm the results described above analyzing whole extracts from Cos-1 cells co-transfected with CK2ß-MycHis and full length Wee1 or one of the Wee1 C-terminal deletion mutants which failed to bind CK2 3 (i.e., Wee1-215*), by gel filtration chromatography (Fig. 4). Full length Wee1 eluted in three major peaks (Fig. 4A) at positions corresponding to molecular masses in the range of 850-1,600 kDa (i.e., fractions 18-20), 230-500 kDa (i.e., fractions 23-25) and 80-130 kDa (i.e., fractions 28-30), respectively. CK2ß-MycHis eluted over a wide range of molecular masses (i.e., fractions 18-30). We then analyzed the elution profile of extracts from cells co-transfected with Wee 1-215* mutant and CK2ß-MycHis (Fig. 4B). Wee $1-215^{*}$ eluted in fractions 30 and 31 while endogenous full length Wee1 was present in fractions corresponding to higher molecular masses (i.e., fractions 27 and 28). The elution profile of CK2ß-MycHis was as in Fig. 4A. Next, we performed immunoprecipitation assays using rabbit polyclonal anti-CK $2 \beta$ and fractions corresponding to the three 
A
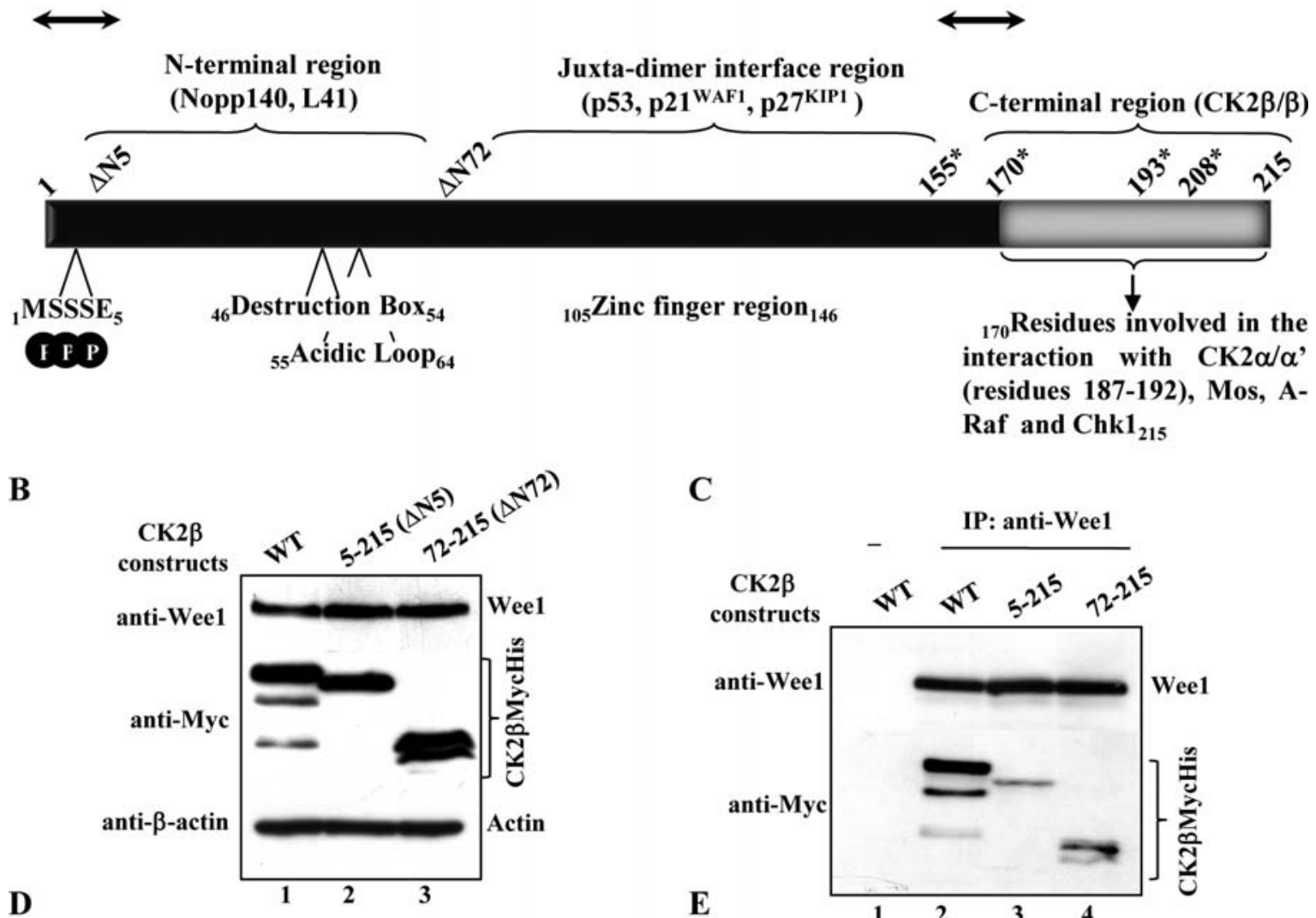

C
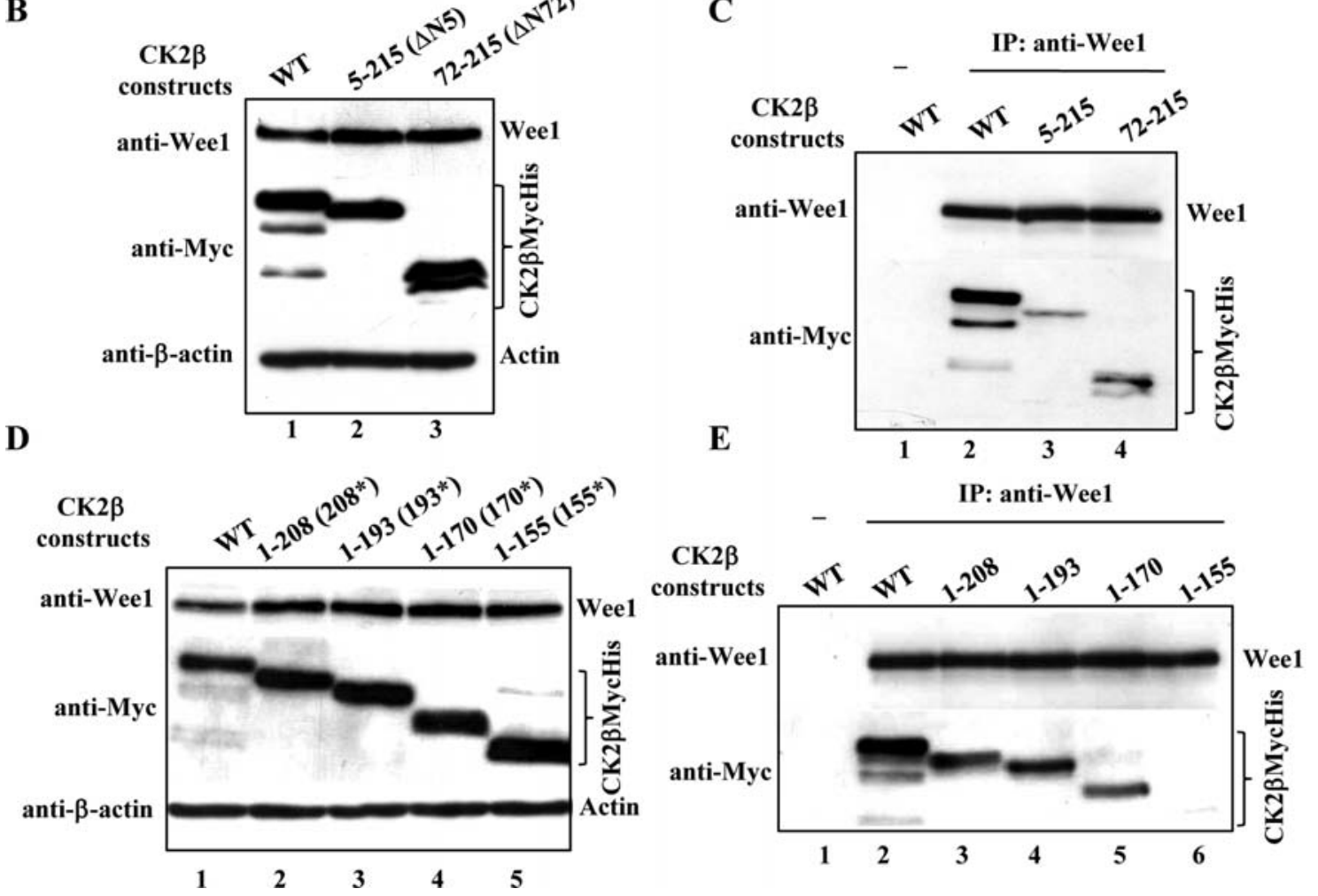

Figure 2. Identification of CK2ß residues required for Wee1 binding. (A) Schematic representation of important features characterizing the CK2ß amino acid sequence. Arrows on top indicate the domains involved in Wee1 binding. (B) Western blot analysis of whole extracts from Cos-1 cells transiently co-transfected with Wee1 and either CK2ß-MycHis (WT, lane 1) or the following N-terminal deletion mutants: CK2ß(5-215)-MycHis (lane 2) and CK2ß(72-215)-MycHis (lane 3) plasmids, respectively. Proteins were detected by employing mouse monoclonal antibodies as indicated in the figure. (C) Whole lysates from cells treated as described in (A) were subjected to immunoprecipitation (IP) in the presence of either control serum (lane 1) or rabbit polyclonal anti-Wee1 (C-20) antibody (lanes 2-4). Immunoprecipitates were subjected to SDS-PAGE and subsequently Western blotting using the indicated mouse monoclonal antibodies. (D) Western blot analysis of whole extracts from cells co-transfected with Wee1 and CK2ß-MycHis (WT, lane 1) or various C-terminal deletion mutants: CK2ß(1-208)-MycHis (lane 2), CK2ß(1-193)-MycHis (lane 3), CK2ß(1-170)-MycHis (lane 4) and CK2ß(1-155)MycHis (lane 5) plasmids, respectively. Detection of proteins was performed as described in (B). (E) Whole lysates from cells treated as indicated in (D) were subjected to immunoprecipitation as described in (C).

elution peaks containing full length Wee1. Co-immunoprecipitation of Wee1 and CK2ß-MycHis or the endogenous CK2 3 was detected in fractions 18-20 indicative for the presence of high molecular mass complexes (Fig. 4C). As expected, no association was observed in immunoprecipitation assays employing fractions resulting from the analysis of protein extract derived from cells co-transfected with Wee1-215* mutant and CK2ß-MycHis (results not shown).
CK2 $2 \beta$ binding to Weel does not influence the Weel-mediated inhibitory phosphorylation of CDK1. Next, we analyzed the functional consequences of the interaction between CK $2 \beta$ and Wee1. We performed in vitro kinase assays employing human recombinant Wee1, active CDK1/cyclin B complex as Wee1 substrate, and increasing amounts of either full length human recombinant $\mathrm{CK} 2 \mathrm{~B}$ (Fig. 5A) or the 1-155 C-terminal deletion mutant (Fig. 5B). Based on results shown in Figs. 2 
A

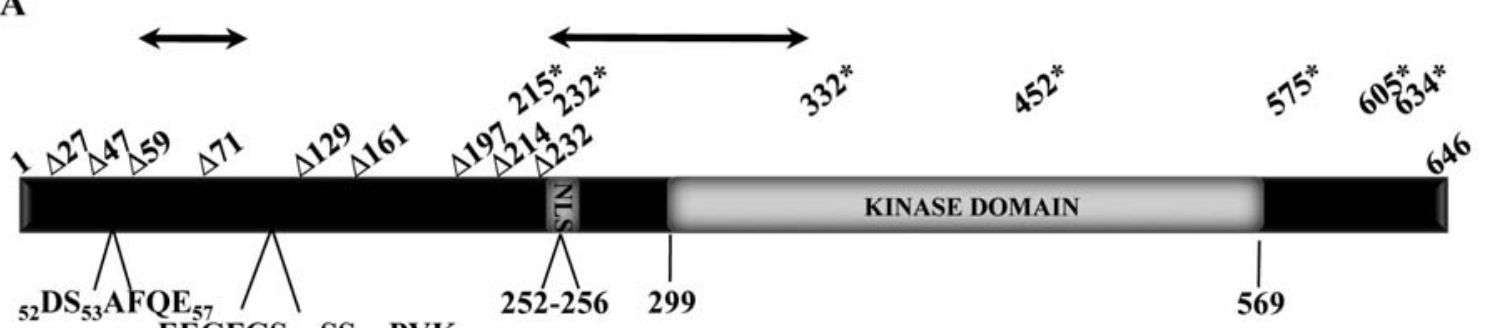

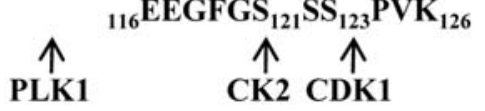

B

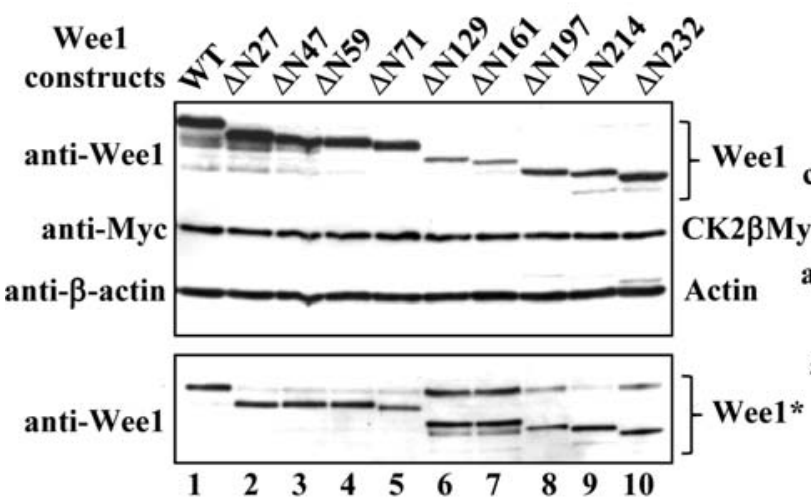

D

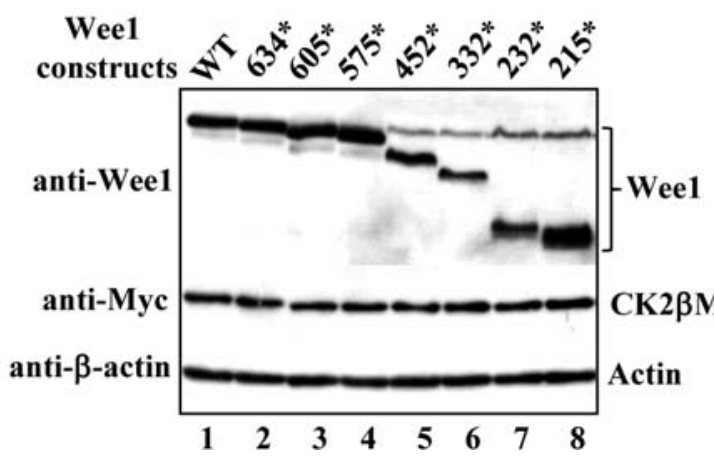

个

Wee1 $_{\text {Y295 }}$ $\uparrow$

Wee1 $_{\text {Y362 }}$
个

Chk1/AKT $_{\mathrm{S} 642}$

C

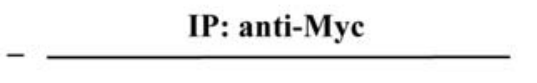

Wee1

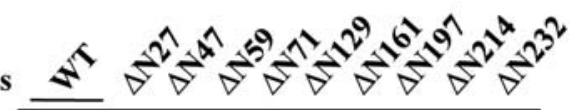
ycHis anti-Wee1

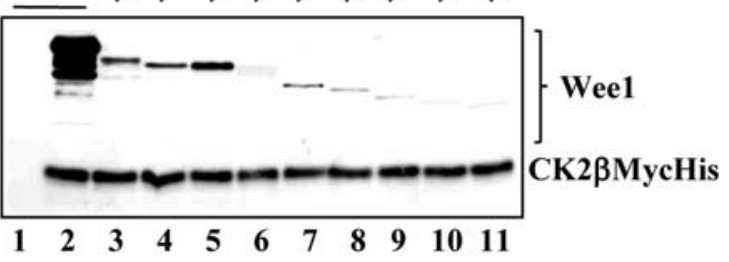

anti-Myc

E

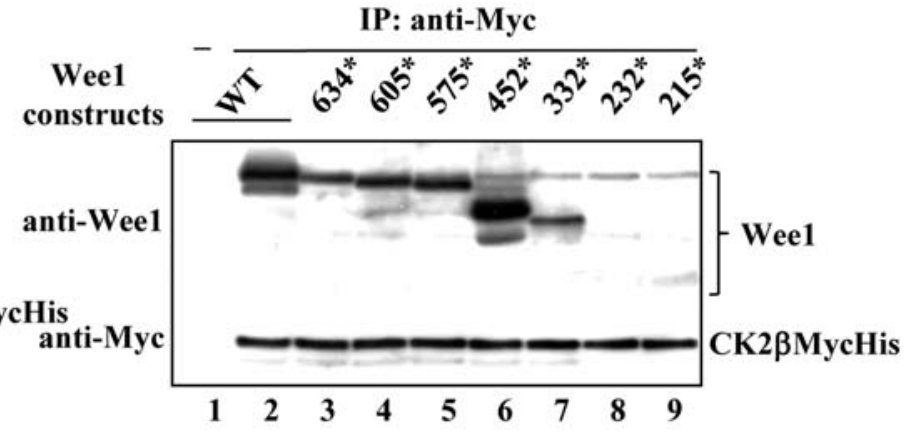

Figure 3. Mapping of the interaction sites of Wee1 with CK2ß. (A) Bar representation of Wee1 amino acid sequence and the residues phosphorylated by the indicated protein kinases that are important for Wee1 activity and/or stability. Arrows on top of the bar indicate the domains involved in the binding with CK2ß. (B) Whole lysates from cells transfected with CK2ß-MycHis and various Wee1 plasmids: WT (lane 1) and N-terminal deletion mutants (lanes 2-10), respectively. Proteins were detected by Western blotting with the indicated mouse monoclonal antibodies. Anti-Wee1 antibody recognizes a Wee1 C-terminal epitope. Wee $1^{*}$ indicates the detection of Wee1 after normalization of protein expression from the various plasmid DNA. (C) Immunoprecipitation was performed as described in Fig. 2C with a rabbit monoclonal anti-Myc antibody. Lane 1 refers to an experiment performed with a control serum. Proteins were detected with the indicated mouse monoclonal antibodies. (D) Whole lysates from cells transfected with CK2B-MycHis and the indicated Wee1 plasmids: WT (lane 1) and various C-terminal deletion mutants (lanes 2-8), respectively. Wee1 was detected with a rabbit polyclonal anti-Wee1 antibody recognizing epitopes in the $\mathrm{N}$-terminal domain of the protein. (E) The immunoprecipitation experiment was performed essentially as described in (C) with a control serum (lane 1) or a mouse monoclonal anti-Myc antibody (lanes 2-9). Proteins were detected with rabbit polyclonal anti-Wee1 and rabbit monoclonal anti-Myc antibodies, respectively.

and 3, we postulated that the presence of CK2ß bound to Wee1 would reverse the Wee1-mediated inhibition of CDK1 activity. The activity of CDK1 was tested by measuring the incorporation of radioactive phosphate into histone $\mathrm{H} 1$. As indicated in Fig. 5A, the presence of Wee1 (lane 5) inhibited the kinase activity of CDK1 about $40 \%$ with respect to the control assay (lane 3). By adding increasing amounts of
CK2ß to the reaction (lanes 6-9) we observed increasing phosphorylation of histone $\mathrm{H} 1$ up to $140 \%$ in the presence of 10 pmol CK2B (lane 9). Moreover, a strong activation of CDK1 was also observed in the presence of 10 pmol CK2 $\beta$ (lane 4), where the CDK1 activity was twice as high as the control assay (lane 3). Wee1-mediated CDK1 phosphorylation levels at the inhibitory Tyr 15 remained unchanged with 


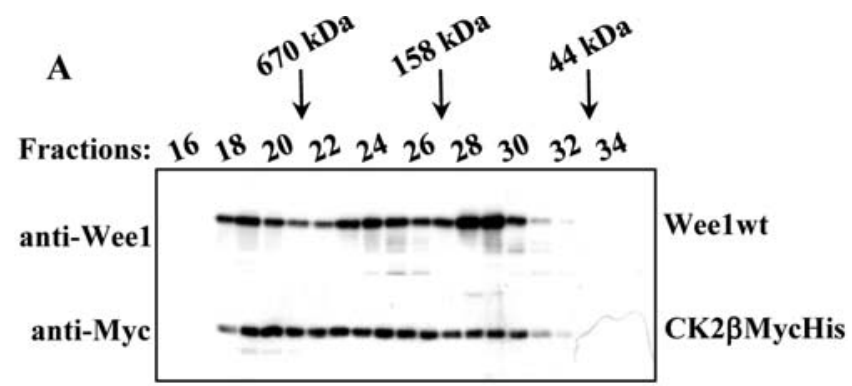

B

Fractions: $16 \begin{array}{lllllllll}18 & 20 & 22 & 24 & 26 & 28 & 30 & 32 & 34\end{array}$

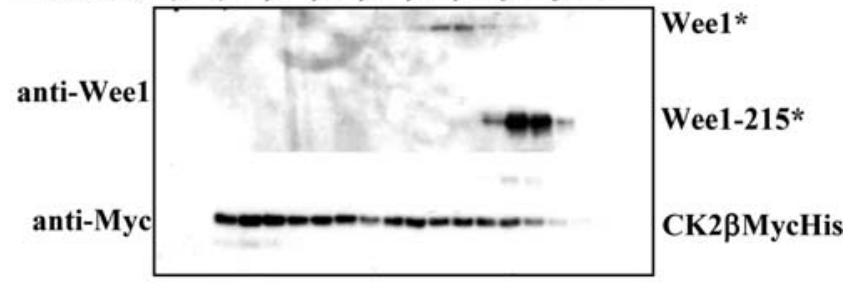

C

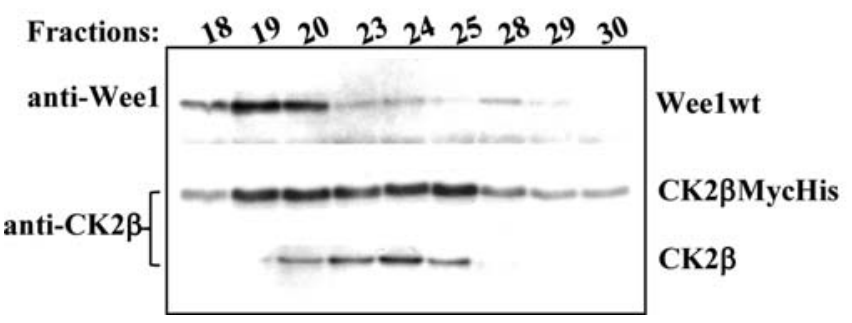

Figure 4. Analytical gel filtration analysis reveals interaction between Weel and CK2B as part of high molecular weight complexes. (A) Whole lysate from cells transfected with wild-type Wee1 and CK2ß-MycHis plasmids was subjected to gel filtration chromatography as described in Materials and methods. The indicated fractions were analyzed by Western blotting employing the indicated mouse monoclonal antibodies. (B) The experiment was carried out as described in (A) except that whole lysate was from cells co-transfected with Wee1-215* C-terminal deletion mutant and CK2ß-MycHis plasmids. Wee $1^{*}$ indicates the detection of endogenous Wee1. (C) The indicated fractions were subjected to immunoprecipitation with a rabbit polyclonal antiCK2 $\beta$ serum. Proteins were detected with the indicated mouse monoclonal antibodies.

respect to the control experiment (lane 5) in the presence of CK2ß (lanes 6-9). Experiments conducted with CK2ß(1-155) deletion mutant which was expected not to reverse the Wee1mediated inhibition of CDK1, led to similar results. Data reported in Fig. 5B, indicate that the association of CK2ß with Wee1 does not affect Wee1-mediated phosphorylation of CDK1 and that the direct interaction between CDK1 and CK2 3 reported in Fig. $5 \mathrm{C}$ might be responsible for the observed upregulation of CDK1 activity in the presence of CK2ß.

\section{Discussion}

Recent evidence has pointed out that the individual subunits of protein kinase may have independent intracellular functions,
A

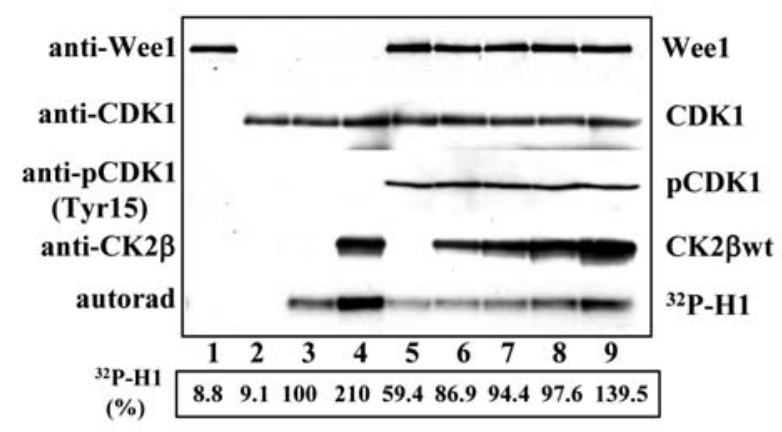

B

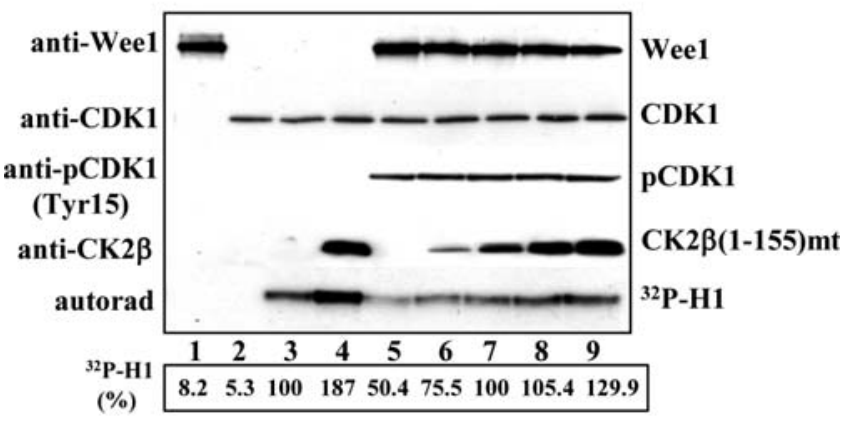

C

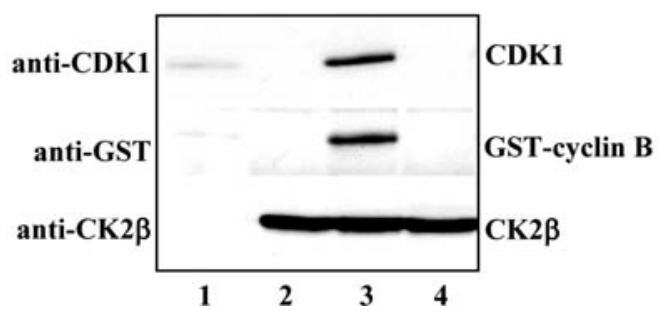

Figure 5. CK2ß binding does not influence the activity of Wee1 with respect to $\mathrm{CDK} 1$ phosphorylation and $\mathrm{CK} 2 \mathrm{~B}$ interacts with $\mathrm{CDK} 1$ and directly upregulates its kinase activity. (A) In vitro kinase assay performed in the presence of 1 pmol recombinant GST-Wee1 (lanes 1 and 5-9), $50 \mathrm{ng}$ CDK1/cylin B (lanes 2-9), $1 \mu \mathrm{g}$ histone $\mathrm{H} 1$ (lanes 1 and 3-9), $1 \mu \mathrm{g}$ GST (lane 2) and increasing amounts of recombinant CK2ßwt (lane 4, 10 pmol; lane 6, 1 pmol; lane 7,2.5 pmol; lane 8, 5 pmol; lane 9, 10 pmol). Proteins were subjected to SDS-PAGE and subsequently Western blot analysis. The phosphorylation of histone $\mathrm{H} 1$ was revealed by autoradiography. The incorporation of radioactive phosphate (expressed in percentage) into the substrate was normalized to the value measured in the control experiment (lane 3). (B) The assay was performed essentially as described in (A) in the presence of increasing amounts of recombinant CK2B(1-155) mutant. (C) Equimolar amounts (15 pmol) of recombinant CK2ß (lanes 2-4), CDK1/cyclinB-GST (lanes 1 and 3), GST (lane 4) were subjected to immunoprecipitation with a rabbit polyclonal anti-CK $2 \beta$ antibody. Immunoprecipitates were subjected to SDS-PAGE and subsequently Western blotting for protein detection by using the indicated antibodies. The detection of GST relative to the control experiment indicated in lane 4 is not shown as no signal was detected.

can be asymmetrically distributed inside the cell and that their expression can be altered in some pathological conditions [e.g., cancer $(1,20)]$. We have recently shown that the regulatory B-subunit of CK2 regulates the stability of Wee1 kinase 
revealing a novel component of the complex machinery that operates at the $\mathrm{G} 2 / \mathrm{M}$ cell cycle transition (16). In the present study, we attempted to: i) closer investigate the nature of the interaction between Wee1 and the CK2 subunits, ii) map the Wee 1 and CK2ß interaction domains and iii) elucidate the functional consequence of their association with respect to Wee1 kinase activity. The usage of recombinant proteins for studying the in vitro interaction between Weel and the individual CK2 subunits revealed that Wee1 predominantly binds $C K 2 \beta$ when present in the reaction assay as individual subunit but not as part of the heterotetrameric enzyme thus suggesting that the CK $2 \beta$ binding regions might be partially masked when CK2ß becomes part of the holoenzyme. The structural mapping for identifying the regions involved in the association with Wee1 led to the interesting observation that not only a C-terminal region located between amino acids 155 and 170 is involved in the binding of Wee 1 but also that the lack of the first CK2ß five amino acids partially abolishes the association between the aforementioned proteins. A number of proteins bind either the C-terminal domain, the juxtadimer interface region or the $\mathrm{N}$-terminal region of $\mathrm{CK} 2 \mathrm{~B}$ (reviewed in refs. 2,21). Interestingly, we found that two CK2ß domains are involved in the association with Wee1. Since the C-terminal region is required for the interaction with the CK2 catalytic subunits and Wee1, this is consistent with the exclusion of CK $2 \alpha$ or $-\alpha^{\prime}$ from the Wee $1 / \mathrm{CK} 2 \beta$ complex that we have previously described (19). Analysis of various Wee1 deletion mutants identified that the two regions between amino acids 59-71 and 232-332 are equally important with respect to the interaction with $C K 2 \beta$ as their deletion completely abolishes the association with CK2B. The region comprising amino acids 232-332 contains a nuclear localization signal (amino acids 252-256), part of the kinase catalytic domain and a Wee1 autophosphorylation site (i.e., Tyr295). By the usage of various deletion mutants we have been able to define the regions involved in the CK2ß/Wee1 association. Since the amino acid sequence spanning from amino acid 232-332 constitutes part of the Wee1 kinase domain we tested whether the presence of CK2ß would affect the kinase activity of Wee1. Results reported in Fig. 5 show that their interaction does not modify the activity of Wee1 with respect to the CDK1 inhibitory phosphorylation at Tyr15. Indeed, we obtained similar results employing a CK2ß deletion mutant unable to bind Wee1. Unexpectedly, we found that the presence of CK2 2 in the reaction mixture competes with Wee1 with respect to CDK1 since CK2 3 which interacts with CDK1, reverts the activity of CDK1 to the levels observed in the control assays. It is noteworthy that in many respects $C K 2 \beta$ is reminiscent of cyclins with respect to regulation of degradation, ligand specificity, formation of complexes and cell-cycle dependent activity (reviewed in ref. 21). While the interaction between Wee 1 and CK2ß is not affecting the activity of Wee1, it cannot be excluded that their association might influence other Wee1 functions such as its cellular localization. We found, in fact, that one of the two regions mediating the interaction with CK2ß comprises the Wee1 nuclear localization signal. It is tempting to speculate, thus, that under specific conditions the association with $C K 2 \beta$ might affect the migration of Wee1 into the nucleus where it exerts full control of CDK1 activity.
The fact that CK2B and Wee1 are part of high molecular mass complexes, as suggested by gel filtration analysis, indicates that the binding of $C K 2 \beta$ might have functional consequences for Wee1 as part of the aforementioned intracellular complexes.

In conclusion, in vitro studies have shown that Wee1 preferentially binds to the regulatory $\beta$-subunit of CK2. By using various deletion mutants we have indentified the regions involved in the association between the afore-mentioned proteins. As their association does not modify Wee1 kinase activity with respect to CDK1 phosphorylation, one of the major substrate targets of Wee1, further work will be decisive to ultimately elucidate the effects of CK2ß binding to Wee1 with respect to regulation of cell cycle progression.

\section{Acknowledgments}

This study was supported by grants to B.G. from the Danish Cancer Society (DP08152) and the Danish Natural Science Research Council (272-07-0258). B.B.O. is supported by a grant from the Danish Cancer Society (DP06083).

\section{References}

1. Litchfield DW: Protein kinase CK2: structure, regulation and role in cellular decisions of life and death. Biochem J 369: 1-15, 2003.

2. Bibby AC and Litchfield DW: The multiple personalities of the regulatory subunit of protein kinase CK2: CK2 dependent and $\mathrm{CK} 2$ independent roles reveal a secret identity for CK2beta. Int J Biol Sci 1: 67-79, 2005.

3. St-Denis NA and Litchfield DW: Protein kinase CK2 in health and disease: from birth to death: the role of protein kinase CK2 in the regulation of cell proliferation and survival. Cell Mol Life Sci 66: 1817-1829, 2009.

4. Lou DY, Dominguez I, Toselli P, Landesman-Bollag E, O'Brien C and Seldin DC: The alpha catalytic subunit of protein kinase CK2 is required for mouse embryonic development. Mol Cell Biol 28: 131-139, 2008.

5. Buchou T, Vernet M, Blond O, Jensen HH, Pointu H, Olsen BB Cochet $\mathrm{C}$, Issinger $\mathrm{OG}$ and Boldyreff $\mathrm{B}$ : Disruption of the regulatory beta subunit of protein kinase $\mathrm{CK} 2$ in mice leads to a cell-autonomous defect and early embryonic lethality. Mol Cell Biol 23: 908-915, 2003.

6. Xu X, Toselli PA, Russell LD and Seldin DC: Globozoospermia in mice lacking the casein kinase II alpha' catalytic subunit. Nat Genet 23: 118-121, 1999.

7. Olsten ME and Litchfield DW: Order or chaos? An evaluation of the regulation of protein kinase CK2. Biochem Cell Biol 82: 681-693, 2004.

8. Meggio F and Pinna LA: One-thousand-and-one substrates of protein kinase CK2? FASEB J 17: 349-368, 2003.

9. Parker LL, Atherton-Fessler S and Piwnica-Worms H: p107wee1 is a dual specificity kinase that phosphorylates $\mathrm{p} 34^{\mathrm{cdc} 2}$ on tyrosine 15 . Proc Natl Acad Sci USA 89: 2917-2921, 1992.

10. Mueller PR, Coleman TR, Kumagai A and Dunphy WG: Myt1: a membrane-associated inhibitory kinase that phosphorylates Cdc2 on both threonine-14 and tyrosine-15. Science 270: 86-90, 1995.

11. Pines J: Four-dimensional control of the cell cycle. Nat Cell Biol 1: E73-E79, 1999.

12. Guerra B, Issinger OG and Wang JY: Modulation of human checkpoint kinase Chk1 by the regulatory beta-subunit of protein kinase CK2. Oncogene 22: 4933-4942, 2003.

13. Kreutzer J and Guerra B: The regulatory beta-subunit of protein kinase CK2 accelerates the degradation of CDC25A phosphatase through the checkpoint kinase Chk1. Int J Oncol 31: 1251-1259, 2007.

14. Watanabe N, Arai H, Nishihara Y, Taniguchi M, Watanabe N, Hunter T and Osada H: M-phase kinases induce phosphodependent ubiquitination of somatic Wee1 by SCFbeta-TrCP. Proc Natl Acad Sci USA 101: 4419-4424, 2004. 
15. Watanabe N, Arai H, Iwasaki J, Shiina M, Ogata K, Hunter T and Osada $\mathrm{H}$ : Cyclin-dependent kinase (CDK) phosphorylation destabilizes somatic Wee1 via multiple pathways. Proc Natl Acad Sci USA 102: 11663-11668, 2005.

16. Yde CW, Olsen BB, Meek D, Watanabe N and Guerra B: The regulatory beta-subunit of protein kinase $\mathrm{CK} 2$ regulates cell-cycle progression at the onset of mitosis. Oncogene 27: 4986-4997, 2008 .

17. Olsen BB, Bjorling-Poulsen M and Guerra B: Emodin negatively affects the phosphoinositide 3-kinase/AKT signaling pathway: a study on its mechanism of action. Int J Biochem Cell Biol 39: 227-237, 2007.

18. Niefind K, Guerra B, Ermakowa I and Issinger OG: Crystal structure of human protein kinase CK2: insights into basic properties of the CK2 holoenzyme. EMBO J 20: 5320-5331, 2001
19. Olsen BB and Guerra B: Ability of CK2beta to selectively regulate cellular protein kinases. Mol Cell Biochem 316: 115-126, 2008.

20. Stalter G, Siemer S, Becht E, Ziegler M, Remberger K and Issinger OG: Asymmetric expression of protein kinase CK2 subunits in human kidney tumors. Biochem Biophys Res Commun 202: 141-147, 1994.

21. Bolanos-Garcia VM, Fernandez-Recio J, Allende JE and Blundell TL: Identifying interaction motifs in CK2beta - a ubiquitous kinase regulatory subunit. Trends Biochem Soc 31: 654-661, 2006. 